\title{
Photoconductivity in highly tetrahedral diamondlike amorphous carbon
}

\author{
G. A. J. Amaratunga, V. S. Veerasamy, W. I. Milne, C. A. Davis, ${ }^{\text {a) }}$ S. R. P. Silva, \\ and H. S. Mackenzie \\ Department of Engineering, Cambridge University, Trumpington Street, \\ Cambridge CB2 1PZ, United Kingdom
}

(Received 21 January 1993; accepted for publication 15 May 1993)

\begin{abstract}
A photoconductive effect is observed in tetrahedral amorphous carbon (ta-C) or amorphous diamond films with a high proportion of $s p^{3}$ bonding and a structure analogous to amorphous silicon. The spectral response is shown to peak at around $750-800 \mathrm{~nm}$ and extend into the near UV region. The maximum photoresponse coincides with the optical absorption edge. The quantum efficiency of $300 \mathrm{~nm}$ thick films with interdigitated electrodes is over $10 \%$ in the wavelength range $450-800 \mathrm{~nm}$.
\end{abstract}

There is presently great interest in hydrogen-free tetrahedral amorphous carbon (ta-C) because of its high diamondlike $s p^{3}$-bonded carbon content $(70 \%-85 \%)$ compared to other amorphous carbon films. This bonding gives ta-C many valuable properties such as high mechanical hardness, low friction, and chemical inertness. ${ }^{1}$ ta-C is also a semiconductor with an optical band gap in the range of $1.65-2.0 \mathrm{eV} .^{2}$ The coupling of optical absorption and space charge limited current flow results have shown the density of states at the valence band edge to be of the order of $10^{21}$ $\mathrm{cm}^{-3} \mathrm{eV}^{-1}$, decreasing exponentially to $10^{18} \mathrm{~cm}^{-3} \mathrm{eV}^{-1}$ at $0.4 \mathrm{eV}$ from the valence band edge. ${ }^{3}$ The ta-C films used in this work were deposited using a cathodic vacuum are and are optically smooth, pin hole free and can be grown over areas up to $10 \mathrm{~cm}^{2}$ at relatively low temperatures $\left(<60^{\circ} \mathrm{C}\right)$. The cathodic arc is a low voltage, high current discharge in which the current is concentrated at one or more micron-sized cathode spots. ${ }^{4}$ The extremely high current densities at the cathode spot cause rapid evaporation of the solid cathode material, most of which is ionized in an intense plasma associated with the cathode spots. In the case of a carbon cathode (ultra-pure graphite), the emitted ions are primarily $\mathrm{C}^{+}$and $\mathrm{C}^{2+}$ ions. ${ }^{5}$ The cathodic arc plasma stream is passed through a magnetic torus to filter out the macroparticles and neutrals. This ensures that only carbon ions impinge on the substrate to form thin films of ta-C. The substrate can be left at floating potential or if conducting can be biased negatively to increase the carbon ion energies. Thin films of ta-C ranging from 100 to 300 $\mathrm{nm}$ in thickness were grown on LPCVD grown silicon nitride and fused quartz substrates under floating potential. Under these conditions, the average energy of the carbon ions is equal to the energy with which they are emitted from the cathode, i.e., about $20 \mathrm{eV}$. A deposition rate of about $3 \mathrm{~nm} / \mathrm{s}$ was achieved with a nominal magnetic field strength of $60 \mathrm{mT}$. The arc voltage and current were $20 \mathrm{~V}$ and $40 \mathrm{~A}$, respectively, during deposition. Structural characterization of the films deposited under these conditions was carried out using a transmission electron microscope (TEM) fitted with an electron energy loss spectroscopy attachment. Diffuse rings typical of amorphous materials

\footnotetext{
a) Permanent address: School of Physies, University of Sydney, NSW 2006, Australia.
}

were observed in the diffraction patterns of the films. A plasmon peak of about $30 \mathrm{eV}$ and an $s p^{3}$-bonded carbon content of approximately $70 \%$ was measured. ${ }^{3}$

Interdigitated gap-cell structures consisting of $100 \mathrm{~nm}$ thick, $5 \mathrm{~mm}$ long gold contacts with spacings of $50 \mu \mathrm{m}$ were patterned on the ta-C films using photolithography. A programmable voltage source was then connected to the gold contacts and the current passing through the ta-C film was monitored using a Keithley picoammeter with a sensitivity of the order of $10^{-13} \mathrm{~A}$. All electrical measurements were carried out in the plane of the films. The current-voltage characteristics of the ta-C gap cells under dark and light conditions are shown in Fig. 1. An AM1 fiber optic light source was used for head-on illumination in both continuous and pulsed modes. Symmetrical $I-V$ curves measured with change in voltage polarity confirmed the ohmic nature of the contacts. The current increase of one to two orders of magnitude when the structures were exposed to light corresponds to a decrease in the resistivity of ta-C films from $10^{7}$ to $10^{5} \Omega \mathrm{cm}$. This is attributed to a photoconductive effect in ta-C films.

The spectral response of the ta-C thin films was monitored over the wavelength range $350-1050 \mathrm{~nm}$. The photoresponse of the interdigitated ta-C photoconductors were

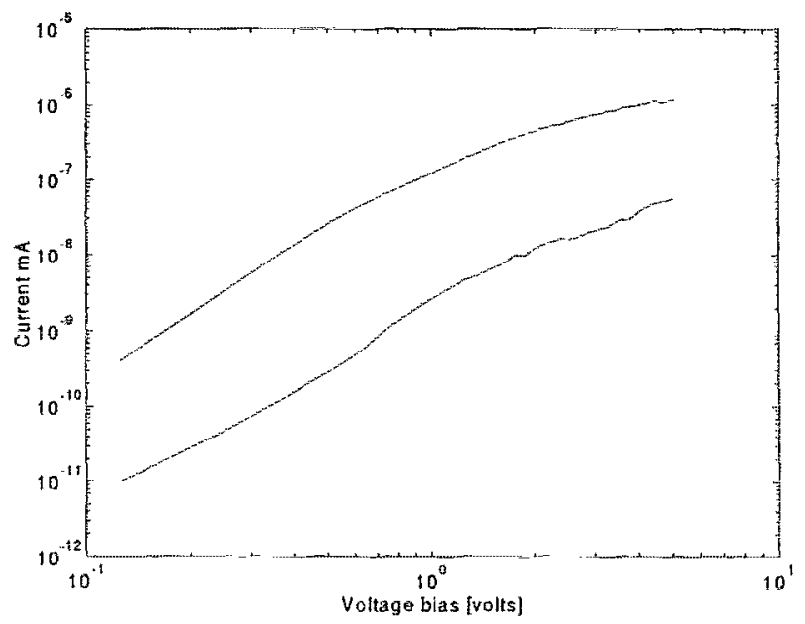

FIG. 1. Current-voltage characteristics of the interdigitated ta-C gap cells in the dark and under white light conditions. 


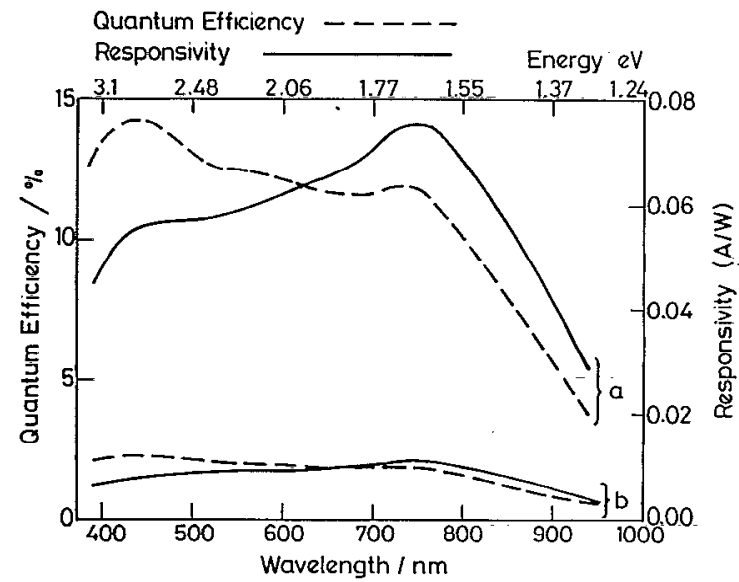

FIG. 2. Quantum efficiency and responsivity for (a) $300 \mathrm{~nm}$ and (b) 100 $\mathrm{nm}$ thick films.

characterized by focusing light in the $350-1050 \mathrm{~nm}$ wavelength range. The source of radiation was a tungsten lamp in conjunction with a spectrometer with 800 line $/ \mathrm{mm}$ gratings blazed at $1 \mu \mathrm{m}$ intervals. The spectral resolution of the system was $5 \mathrm{~nm}$. The responsivity and external quantum efficiency of the ta-C photoconductor was obtained by comparison with a standard of known responsivity, a super UV series 7 photodiode. Satisfactory agreement was obtained between the average photodiode calibration curve provided by the manufacturer and the responsivity calibration on the specific diode done in our laboratory. The spectral response curves for films with thicknesses 100 and 300 $\mathrm{nm}$ are shown in Fig. 2. The incident light was chopped at $100 \mathrm{~Hz}$ and the ac component of the photocurrent response was measured using a lock-in amplifier.

The external quantum efficiency versus wavelength curves for the two samples of different thicknesses are also shown on Fig. 2. The illuminated areas of the photoconductor and the silicon diode used for calibration were kept constant, equal to $8 \mathrm{~mm}^{2}$. Noteworthy features of the responsivity versus wavelength curves are:

(i) The responsivity curves for both samples show a pronounced peak at about $700-800 \mathrm{~nm}$, corresponding to photon energies between 1.6 and $1.8 \mathrm{eV}$;

(ii) the photoresponse extends into the blue-green region of the spectrum;

(iii) the quantum efficiency of the $300 \mathrm{~nm}$ thick ta-C films lies between $10 \%$ and $12.5 \%$ in the $450-800 \mathrm{~nm}$ wavelength range. The optical band gap of the ta-C deposited on fused quartz is determined to be in the range of $1.8-2.2 \mathrm{eV}$ from the Tauc plot of the transmission spectrum. The maximum photoresponse at $750-800 \mathrm{~nm}$ is found to coincide with the absorption edge. The value of the absorption coefficient $\alpha$ for the films is greater than $10^{4}$ $\mathrm{cm}^{-1}$ from 750 to $400 \mathrm{~nm}$, Fig. 3 .

The current activation energy of the films was also monitored in the dark and under white light. The dark conductivity as a function of temperature is shown in Fig. 4. The dark conductivity $\sigma_{d}$ increases with increasing temperature following the Mott-Twose relationship $\sigma_{d}$ $=\sigma(0) \exp \left(-E_{a} / k T\right)$ suggesting activation of carriers

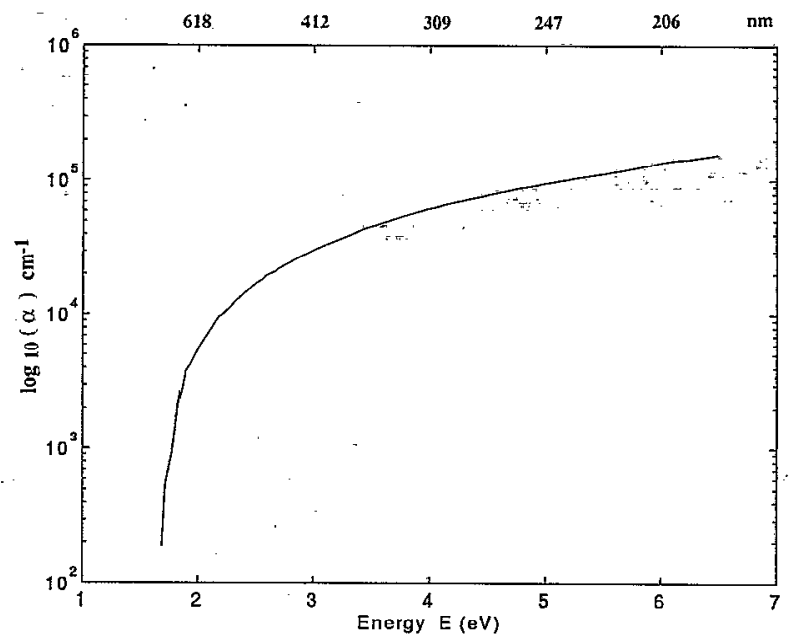

FIG. 3. Optical absorption coefficient for a ta-C film on quartz.

into the extended states. This gives an activation energy $E_{a}$ of $0.2-0.25 \mathrm{eV}$ above the valence band, if one assumes that the as-deposited ta-C is weakly $p$ type. ${ }^{3}$ On the other hand, the variation of conductivity under light was found to be independent of temperature, curve (b) in Fig. 3. This result is in accordance with conductivity under light $\sigma_{\mathrm{ph}}$ being dominated by the generation term in the expression $\sigma_{\mathrm{ph}}=\sigma_{d}+\mu_{0} G \tau$, thus showing that the mobility lifctimc $\left(\mu_{0} \tau\right)$ product is temperature independent (in the range considered) for a given bulk photogeneration rate $G$ in ta-C films.

In the interpretation of the photoconductive results it is reasonable to assume that the photogenerated current is predominantly carried by electrons in the extended states of the conduction band. In this scheme electrons are excited from the extended states in the valence band or from filled localized tail statcs near the valence band edge. The spectral response of photoconductivity, therefore, reveals information regarding the electronic band gap (mobility gap) of this amorphous semiconductor since the mobility gap should be larger than the optical band gap. The occurrence of a shoulder in the responsivity at photon energies in

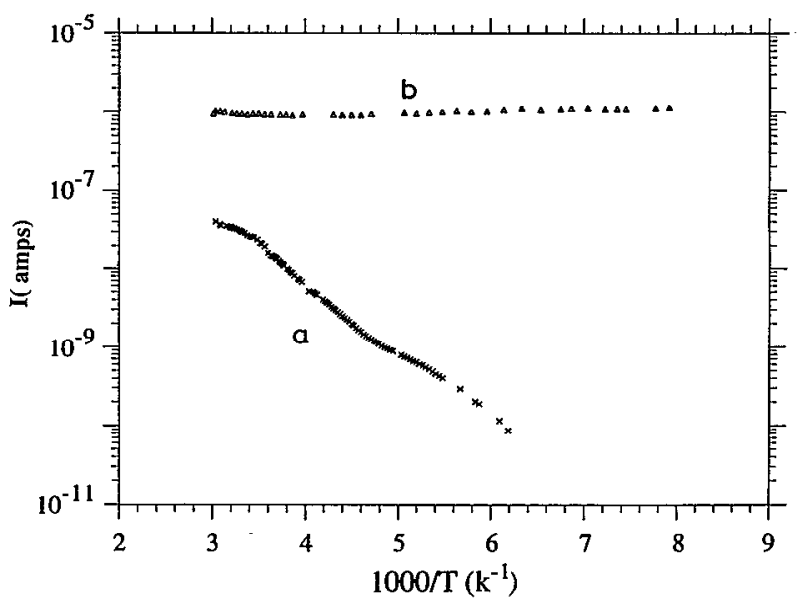

FIG. 4. Variation of (a) dark and (b) under light conductivities of the $300 \mathrm{~nm}$ film with temperature. 
the region of $1.65 \mathrm{eV}(750 \mathrm{~nm})$ agrees well with the analysis of optical properties of these noncrystalline films. On the assumption of parabolic bands and the density of states being a linear function of energy, ${ }^{6,7}$ the expression $(\alpha h v)^{1 / 2}=\left(h v-E_{0}\right)$ leads to a value of $1.6-1.8 \mathrm{eV}$ for the optical band gap $E_{0}$, consistent with the responsivity data. However, if the density of states is taken to be a squareroot function of energy, ${ }^{8.9}$ the expression $(n \alpha)^{1 / 2} h v$ $=B\left(h v-E_{0}\right)$ with $n$ taken as the refractive index yields an optical band gap $E_{0}$ between 2 and $2.2 \mathrm{eV}$ for ta-C films, similar to that reported by Lossy et al. ${ }^{10}$ using the same procedure. The photoconductivity results are, therefore, consistent with the optical absorption data if the density of states is taken to be a linear function of energy.

In summary, hydrogen-free ta- $\mathrm{C}$ films deposited from a filtered vacuum arc source display photoconductivity. The spectral response of the thin films shows that this material has an electronic band gap equal to or greater than $1.6 \mathrm{eV}$. The interdigitated photoconductors are found to have a quantum efficiency of over $10 \%$ in the wavelength range
$400-750 \mathrm{~nm}$. The blue light sensitivity of ta-C makes its use as a complementary material to $a-\mathrm{Si}: \mathrm{H}$ in photodetectors an interesting prospect.

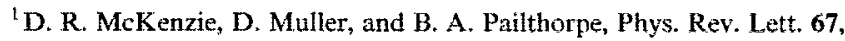
773 (1991).

${ }^{2}$ J. C. Angus, P. Koidl, and S. Domitz, in Plasma Deposited Thin Films, edited by E. Jansen (CRC, Boca Raton, FL, 1986), p. 530.

${ }^{3}$ V. S. Veerasamy, G. A. J. Amaratunga, W. I. Milne, P. Hewill, P. J. Fallon, D. R. McKenzie, and C. A. Davis, Diamond Rel. Mater. 2, 782 (1993).

${ }^{4}$ J. M. Lafferty Vactum Arcs (Wiley, New York, 1980).

${ }^{5}$ V. S. Veerasamy, G. A. J. Amaratunga, and W, I. Milne, IEEE Trans. Plasma Sci. (to be published).

${ }^{\circ}$ E. A. Davis and N. F. Mott, Philos. Mag. 22, 903 (1970).

${ }^{7}$ N. F. Mott and E. A. Davis, Electronic Processes in Non-Crystalline Materials (Clarendon, Oxford, England 1971), p. 248.

${ }^{8} \mathrm{~J}$. Tauc, in Amorphous and Liquid Semiconductors, edited by J. Tauc (Plenum, London, 1974), p. 159.

${ }^{9} \mathrm{G}$. A. N. Connell, in Amorphous Semiconductors, edited by M. H. Brodsky (Springer, Berlin, 1984), Vol. 56, p. 61.

${ }^{10}$ R. Lossy, D. L. Pappas, R. A. Roy, J. J. Cuomo, and V. M. Sura, Appl, Phys. Lett. 61, 171 (1992). 